\title{
Etched ring absorbing waveguide filter based on a slotted waveguide antenna response
}

\author{
Tinus Stander and Petrie Meyer \\ Department of E\&E Engineering \\ University of Stellenbosch \\ Private Bag X1 \\ 7602 Matieland \\ South Africa \\ Corresponding author: \\ tstander@sun.ac.za \\ Fax: +27218084981
}

\begin{abstract}
A cascade of resistively loaded etched rings in waveguide is proposed to act as the load in a balanced FMCW Reflected Power Canceller topology using a slotted waveguide array antenna. A synthesis technique using derived equivalent circuit models, is presented and tested.
\end{abstract}

Keywords: "absorbing filter", "slotted waveguide antenna", "reflected power canceller", "lossy ring resonator", "circuit model"

Introduction: FMCW radar reflected power cancellers (RPCs) have for many years relied on closed-loop controllers for active cancellation [1]. Recently, a passive balanced topology to cancel transmitter leakage and antenna reflections, was suggested for MMIC implementation [2]. With this topology, cancellation can be achieved across wide bandwidths, but the implementation relies on an accurate dummy load to balance the input reflection of the radar antenna. The implementation of such a load is no trivial task, as the input impedance has to match that of the antenna very closely over the whole band of interest. In this paper, it is shown that the slotted waveguide antenna, often used in high frequency FMCW radars [1] [3], allows for a particularly interesting implementation of such a system, as a load can be designed which approximates the input impedance of the antenna very closely. By periodic cascading of some lossy microwave structure in waveguide, each having transmission and reflection characteristics similar to a single radiating slot of the antenna, a load is obtained which is not only similar to the antenna in construction, but also in input impedance.

A structure suitable for this task was proposed by Mias [4] as a lumped element loaded etched FSS ring. These resistor-loaded rings are shown, in this paper, to have remarkably similar electrical characteristics to longitudinal waveguide slots, making them a natural choice for the problem at hand. This paper presents the design of a waveguide load for the balanced FMCW RPCs system, using the loaded etched FSS rings in waveguide proposed by Mias. An accurate circuit model and design guidelines are derived for the ring 
structure. As an example, a three-element cascaded loaded resonator structure is implemented and tested.

The loaded FSS ring in waveguide: The basic structure of the lumped element loaded etched ring resonator is shown in Fig. 1. Mias originally proposed a double ring implementation [4], but the reflection from such a discontinuity is significantly larger than that obtained from a typical waveguide radiating slot. To reduce the structure's reflection coefficient, the two rings are replaced here by a single ring.

The circuit model of the loaded resonator is shown in Fig. 2. It is based on the model provided in [5], where it is applied to split ring resonators in coplanar waveguide. The model has been adapted to the current implementation by replacing the series coupling in [5] with shunt coupling, simplifying the model by replacing the mutually coupled inductors with an ideal transformer, adding a shunt capacitor to model the substrate in waveguide [6], and adding resistance to the resonant ring. The shunt admittance $y$ of the ring itself (neglecting the transmission lines and shunt capacitor $C_{p}$ ) is expressed as

$$
y(\omega)=g(\omega)+j b(\omega)=\frac{n^{2} R_{r}}{\left(\omega L_{r}-\frac{1}{\omega C_{r}}\right)^{2}+R_{r}^{2}}+j\left(\frac{n^{2}\left(\frac{1}{\omega C_{r}}-\omega L_{r}\right)}{\left(\omega L_{r}-\frac{1}{\omega C_{r}}\right)^{2}+R_{r}^{2}}\right)
$$

In order to calculate element values for the equivalent circuit, the ring structure is simulated in a numerical EM solver, in this case, CST Microwave Studio. Using the simulated values of peak conductance and the susceptance slope at the centre frequency, and choosing $R_{r}$ to be the physical resistance in the structure $2 r$ (where $r$ is the value of each of the two physical resistors), values for $L_{r}, C_{r}$ and $n$ can easily be derived. Applying this method to the structure shown in Fig. 1, using dimensions from Table 1 column (a), close correspondence in electrical characteristics between the circuit model and the ring structure is achieved, as shown in Fig. 3.

For the purpose of this paper, it is important to investigate the ability of the loaded resonator structure to approximate the electrical characteristics of the waveguide radiating slot. This is also illustrated in Fig. 3, where the conductance and susceptance (calculated from simulated S-parameters) of a longitudinal waveguide slot $29.4 \mathrm{~mm}$ in length with $4.0 \mathrm{~mm}$ offset is added to the comparison. From this graph, it is clear that the slot and the loaded resonator can be designed to display very similar electrical characteristics, both represented well by the given circuit model.

Parametric study of loaded resonator: This section of the paper will describe, in broad terms, the relationships between the element dimensions shown in Fig. 1, and the circuit elements shown in Fig. 2. This is included to aid the design of the loaded resonator for specific values of peak conductance, centre frequency, or susceptance slope at centre frequency. Exact values are best determined by iterative simulation. In all cases, the different values of the 
varied parameter are indicated in the figure's legend. All other dimensions are as indicated in Table 1, column (a).

The ring inductance $L_{r}$ and capacitance $C_{r}$ in the circuit model determines the resonant frequency of the circuit. In the loaded resonator, altering the resonant frequency is achieved primarily by varying the path length around the ring by adjusting $d_{x}$, as shown in Fig. 4 . The inductance $L_{r}$ can also be controlled (to a lesser extent) with the width of the etched ring, as that influences the impedance of the line. A narrower track has more inductance, causing a marginally lower resonant frequency and steeper slope.

Also noticeable from Fig. 4 is a change in peak resonant conductance $g\left(\omega_{0}\right)$ as a function of path length. This circuit effect is achieved by reducing the transformer ratio $n$. Physically, reducing the path length reduces the flux linkage to the ring. With only the $T E_{10}$ field component normally incident, the flux linkage is calculated as

$$
\lambda_{f}=-2\left(d_{y}-w\right) \mu_{0} A_{10} \frac{a}{\pi} \sin \left(\frac{\pi\left(d_{x}-w\right)}{a}\right)
$$

From (2), it can be seen that $\lambda_{f}$ is linearly dependent on $d_{y}$, but sinusoidally dependent on $d_{x}$. For values of $d_{x} \approx a / 2$ (as is the case here), the sinusoidal term would be close to a stationary point, and variations in $d_{x}$ would result in much smaller changes in $g\left(\omega_{0}\right)$ than variations in $d_{y}$. The sensitivity of the $g\left(\omega_{0}\right)$ to variation in $d_{y}$ is illustrated in Fig. 5.

Also interesting to note, is that, if the ring is offset from the middle of the waveguide ( $y z$-plane) by a distance $x_{0}, \lambda_{f}$ is expressed as

$$
\lambda_{f}=2\left(d_{y}-w\right) \mu_{0} A_{10} \frac{a}{\pi} \cos \left(\frac{\pi\left(a+2 x_{0}\right)}{a}\right) \sin \left(\frac{\pi\left(d_{x}-w\right)}{a}\right)
$$

This indicates that $g\left(\omega_{0}\right)$ can be reduced further by offsetting the ring to the left or right of the waveguide. This is illustrated in Fig. 6. This further mechanism to reduce $g\left(\omega_{0}\right)$ can be important, as typical values of $g\left(\omega_{0}\right)$ required to approximate a radiating slot can be quite low.

The capacitance $C_{p}$ models the effect of the substrate in the loaded resonator. It therefore stands to reason that increasing the substrate's thickness and surface area (by reducing $C_{x}$ and $c_{y}$ ) would increase the value of $C_{p}$ and the shunt susceptance $b$. Since there is no substrate involved in the waveguide slot, it is required that the substrate have as little effect in the loaded resonator as possible, if the waveguide slot is to be approximated accurately. To this end, 5 mil thickness substrate was used in prototyping, and sections of substrate were cut away.

Three-element example: To verify the proposed design process, a matched load was designed, manufactured and measured for an arbitrary set of 3 cascaded longitudinal slots in WR-90 waveguide, as shown in Fig. 7.

Initially, the three slots were individually simulated in CST Microwave Studio, using the dimensions in Table 2. From this, the electrical characteristics $\omega_{0}$, 
$g\left(\omega_{0}\right)$ and $\partial b /\left.\partial \omega\right|_{(\omega=\omega 0)}$ of each slot were determined, and parameters for three equivalent circuit representations calculated using (1). Next, these calculated circuit values where entered in a single circuit representation of the three slot cascaded structure, as shown in Fig. 8, using initial values of $d_{12}=d_{23}=d_{s}$.

The full structure shown in Fig. 7 was then simulated, and the port parameters $\left|S_{11(\mathrm{~s})}\right|$ and $\left|S_{21(\mathrm{~s})}\right|$ entered as a goal function in a optimisation of the circuit in Fig. 8, where the previously calculated circuit values were used as initial values. This optimisation step, performed using AWR Microwave Office, is necessitated by the presence of external mutual coupling between longitudinal waveguide slots [7], which is neither modelled by the circuit representation, nor occurs between adjacent loaded ring resonators. Since the problem is underconstrained (i.e, multiple sets of circuit values rendering identical responses) the values $C_{p}$ and $R_{r}$ are chosen as fixed.

From the optimised circuit, the new values $\omega_{0}, g\left(\omega_{0}\right)$ and $\partial b /\left.\partial \omega\right|_{(\omega=\omega 0)}$ of each of the three cascaded circuit elements were recorded, and three loaded resonators designed to have a similar electrical response using the techniques discussed previously. Note that, to match the value of $\partial b /\left.\partial \omega\right|_{(\omega=\omega 0)}$ accurately between the loaded resonator and the circuit, it is necessary to change the value of the SMD resistor in the EM simulation slightly so that $R_{r} \neq$ 2 r.

Following this design procedure, the dimensions of loaded resonators 1,2 and 3 were obtained as indicated in Table 1, columns (b), (c) and (d). Not indicated in the table is the optimised waveguide length between resonators 1 and $2\left(I_{12}\right)$ of $27.24 \mathrm{~mm}$ and between resonators 2 and $3\left(I_{23}\right)$ of $26.03 \mathrm{~mm}$.

Measurement results: A three-ring prototype was constructed using Taconic TLY-5A substrate and 0402 size thin film resistors. The measured reflection response, compared to the reflection responses of the circuit model and slot array, is shown in Fig. 9, with the difference in slot array and ring array response shown in Fig. 10. A reflection match of $-15 \mathrm{~dB}$ is achieved across the entire X-band.

Conclusion: This paper presents a technique to design an accurate matched load to a longitudinally slotted waveguide antenna array by cascading loaded etched ring resonators in waveguide. The generalised synthesis procedure is based on a one-to-one correspondence between loaded resonating rings and waveguide slots, using an intermediate circuit model representation. A prototype load is shown to match an 3 slot array to within $-15 \mathrm{~dB}$ across the X-band.

Acknowledgements: The authors would like to thank CST GmbH for the use of CST Microwave Studio 2006 and 2006B in preparing this paper, as well as Applied Wave Research Inc. for the use of AWR Microwave Office 2006 (MWO-225). 
References:

1. P. D. L. Beasley, A. G. Stove, B. J. Reits, and B. O. As, Solving the problems of a single antenna frequency modulated $\mathrm{CW}$ radar, IEEE International Radar Conference (1990), 391-395.

2. J. G. Kim, S. Ko, S. Jeon, J. W. Park, and S. Hong, Balanced topology to cancel TX leakage in CW radar, IEEE Microwave and Wireless Components Letters 14 (2004), 443-445.

3. R. E. Collin, Antennas and Radiowave Propagation, McGraw-Hill, 1985.

4. C. Mias, Frequency selective absorption using lumped element frequency selective surfaces, Electronics Letters 39 (2003), 847-849.

5. F. Martin, J. Bonache, F. Falcone, M. Sorolla, and R. Marques, Split ring resonator-based left-handed coplanar waveguide, Applied Physics Letters 83 (2003), 4652-4654.

6. A. J. Kelly, The negative capacitor, an impedance matching element for dielectric-filled transmission line, IEEE Trans Microwave Theory and Tech 15 (1967), 583-584.

7. R. S. Elliot and L. A. Kurz, The Design of Small Slot Arrays, IEEE Trans. Antennas Propagat., AP-26 (1978), 214-219. 
Figure 1:

Loaded etched ring resonator with basic dimensions. Not indicated in the diagram is the track etch thickness $t$ and the chip resistor's value $r$.

Figure 2:

Equivalent circuit model of loaded resonator.

Figure 3:

Comparison of circuit model admittance to data extracted from EM analysis.

Figure 4:

Variation of resonant frequency and peak conductance as a function of $d_{x}$ for $d_{y}=1.28$.

Figure 5:

Variation of peak conductance as a function of $d_{y}$ for $d_{x}=12.96$.

Figure 6:

Variation of peak conductance as a function of offset from centre $x_{0}$.

Figure 7:

Cascaded longitudinal waveguide slot array.

Figure 8:

Circuit model representation of cascaded longitudinal waveguide slot array.

Figure 9:

Input reflection of simulated slot array, optimised circuit model and measured loaded resonator structure.

Figure. 10:

Difference in reflection response between simulated slot array and measured loaded resonator structure. 


\begin{tabular}{|l|l|l|l|l|}
\hline $\begin{array}{l}\text { Dimension } \\
\text { (mm, unless indicated otherwise) }\end{array}$ & $(\mathrm{a})$ & $(\mathrm{b})$ & $(\mathrm{c})$ & $(\mathrm{d})$ \\
\hline$a$ (waveguide width) & 22.86 & 22.86 & 22.86 & 22.86 \\
\hline$b$ (waveguide height) & 10.16 & 10.16 & 10.16 & 10.16 \\
\hline$c_{X}$ (substrate cut-away width) & 20 & 20 & 20 & 20 \\
\hline$c_{Y}$ (substrate cut-away depth) & 3.99 & 3.74 & 3.40 & 3.45 \\
\hline$d_{X}$ (ring width) & 12.96 & 12.99 & 12.89 & 13.37 \\
\hline$d_{Y}$ (ring height) & 1.28 & 2.08 & 2.76 & 2.77 \\
\hline$I_{r}$ (resistor length) & 0.51 & 0.25 & 0.25 & 0.25 \\
\hline$w$ (track width) & 0.3 & 0.3 & 0.3 & 0.3 \\
\hline$h$ (substrate height) & 0.13 & 0.13 & 0.13 & 0.13 \\
\hline$t$ (track etch thickness) & 0.035 & 0.035 & 0.035 & 0.035 \\
\hline$r$ (chip resistor value) $(\Omega)$ & 20 & 20 & 18 & 20 \\
\hline
\end{tabular}




\begin{tabular}{|l|l|}
\hline Dimension & Value $(\mathrm{mm})$ \\
\hline$a$ (waveguide width) & 22.86 \\
\hline$b$ (waveguide height) & 10.16 \\
\hline$d_{s}$ (slot spacing) & 26 \\
\hline$s_{1}$ (slot 1 length) & 15.35 \\
\hline$s_{2}$ (slot 2 length) & 16.30 \\
\hline$s_{3}$ (slot 3 length) & 16.60 \\
\hline$d_{1}$ (slot 1 offset) & 5.49 \\
\hline$d_{2}$ (slot 2 offset) & 5.42 \\
\hline$d_{3}$ (slot 3 offset) & 5.38 \\
\hline
\end{tabular}




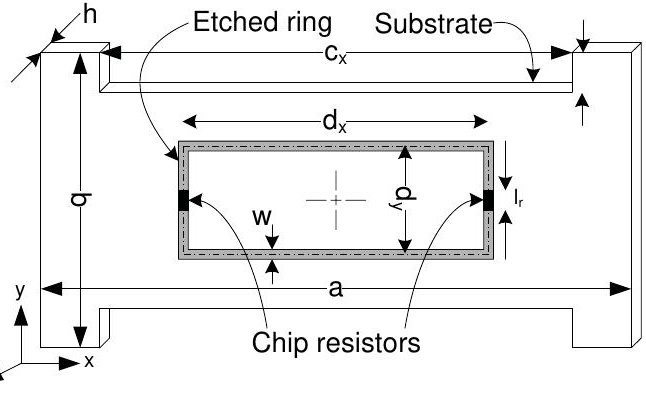




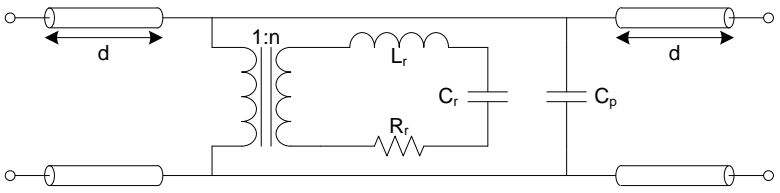




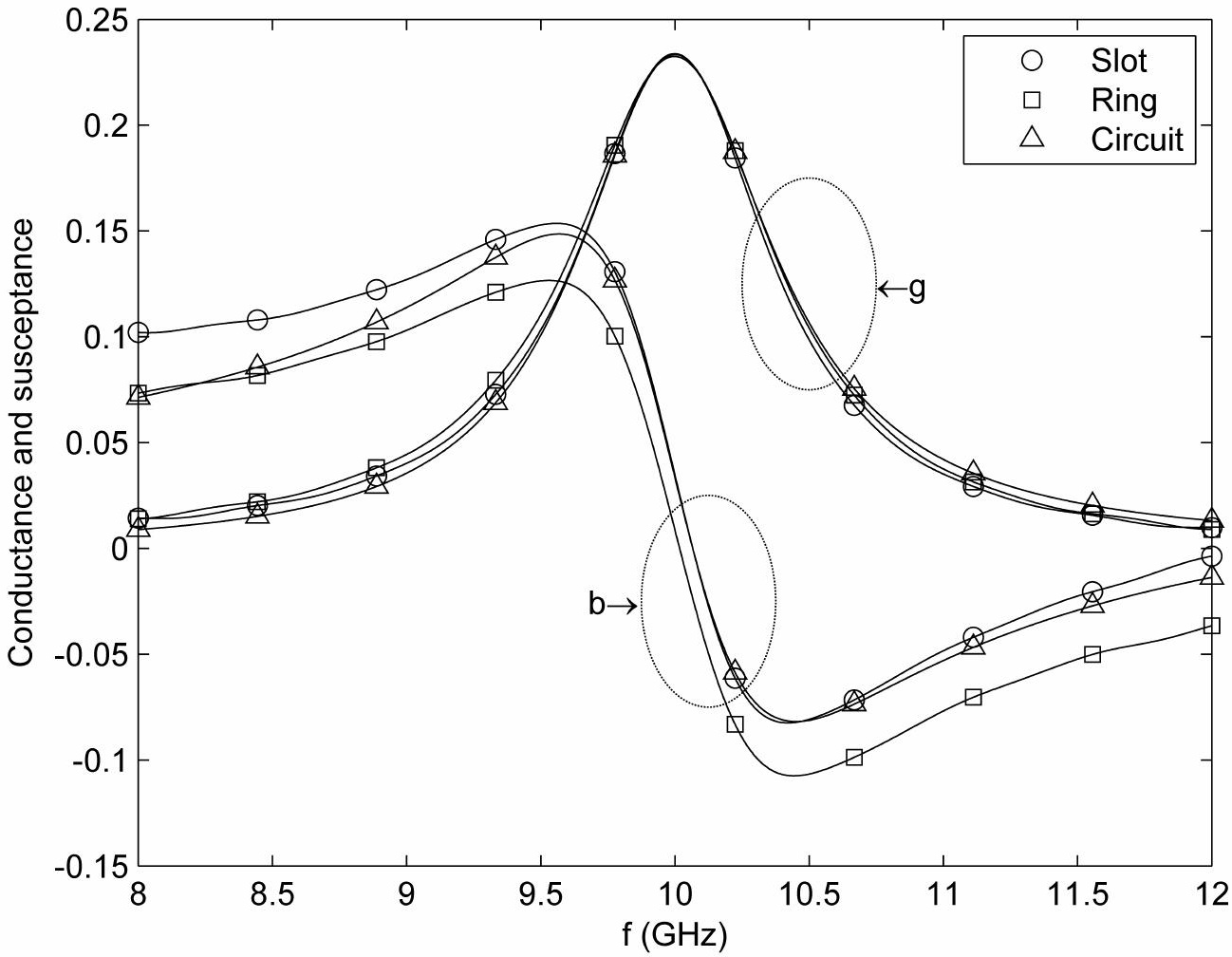




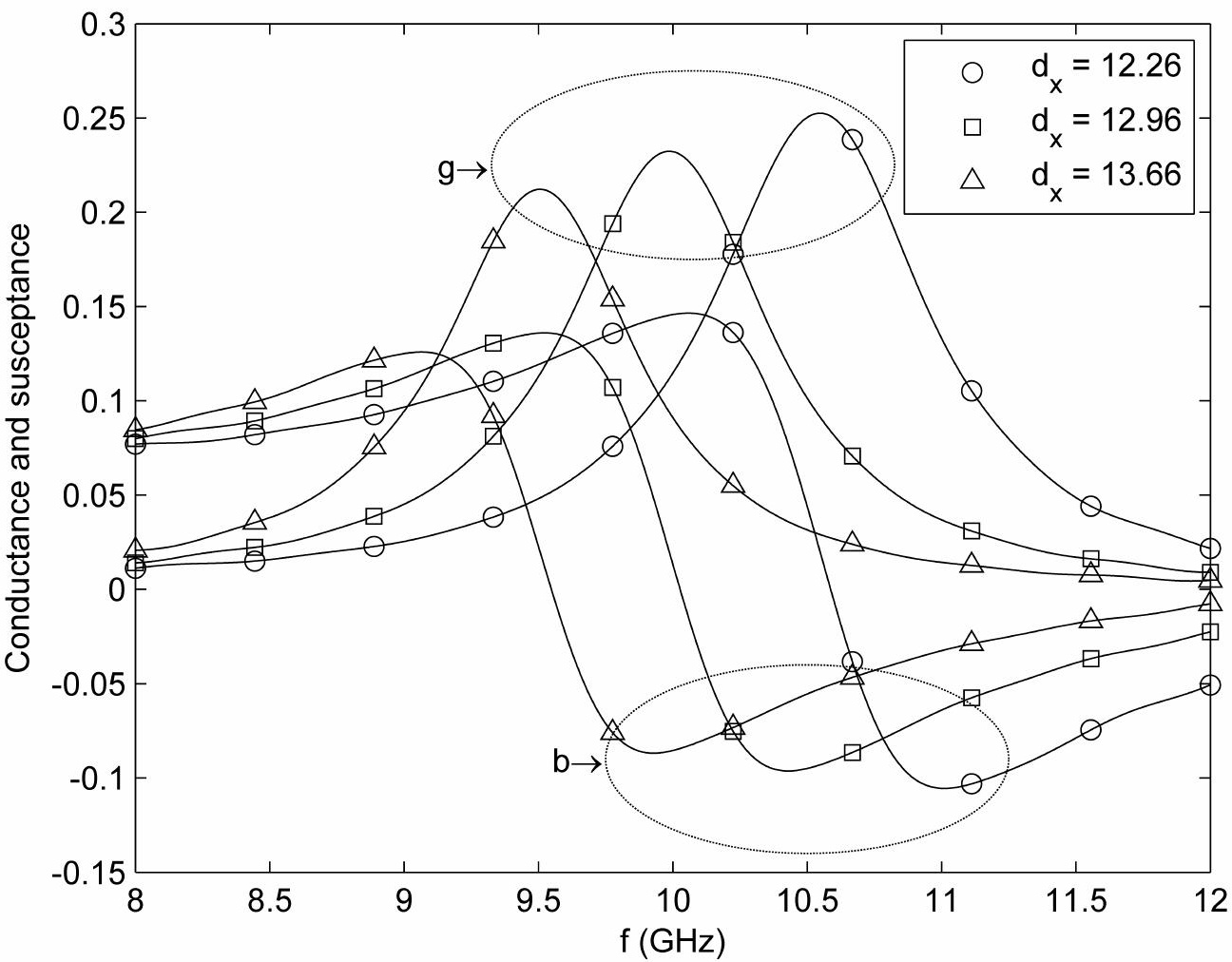




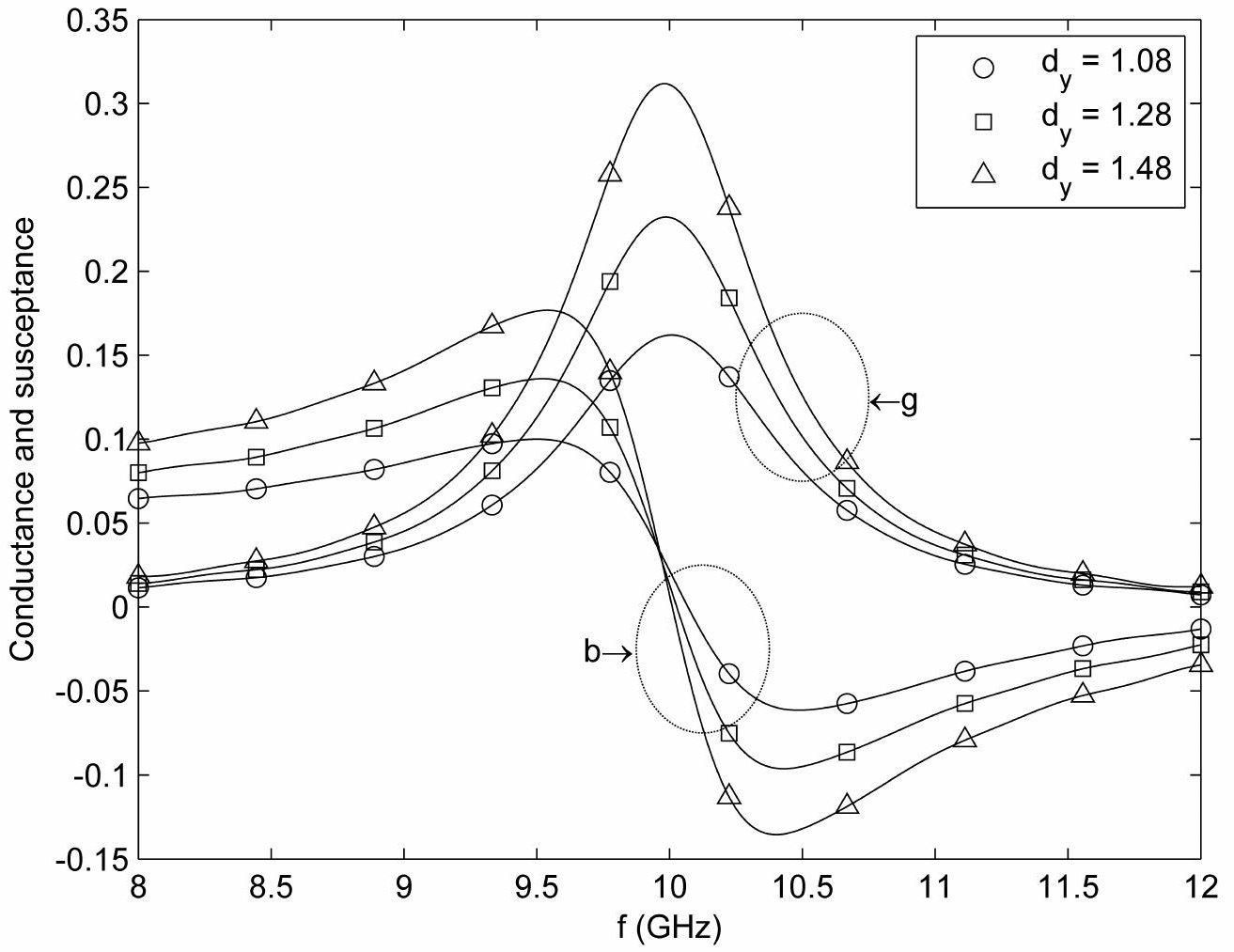




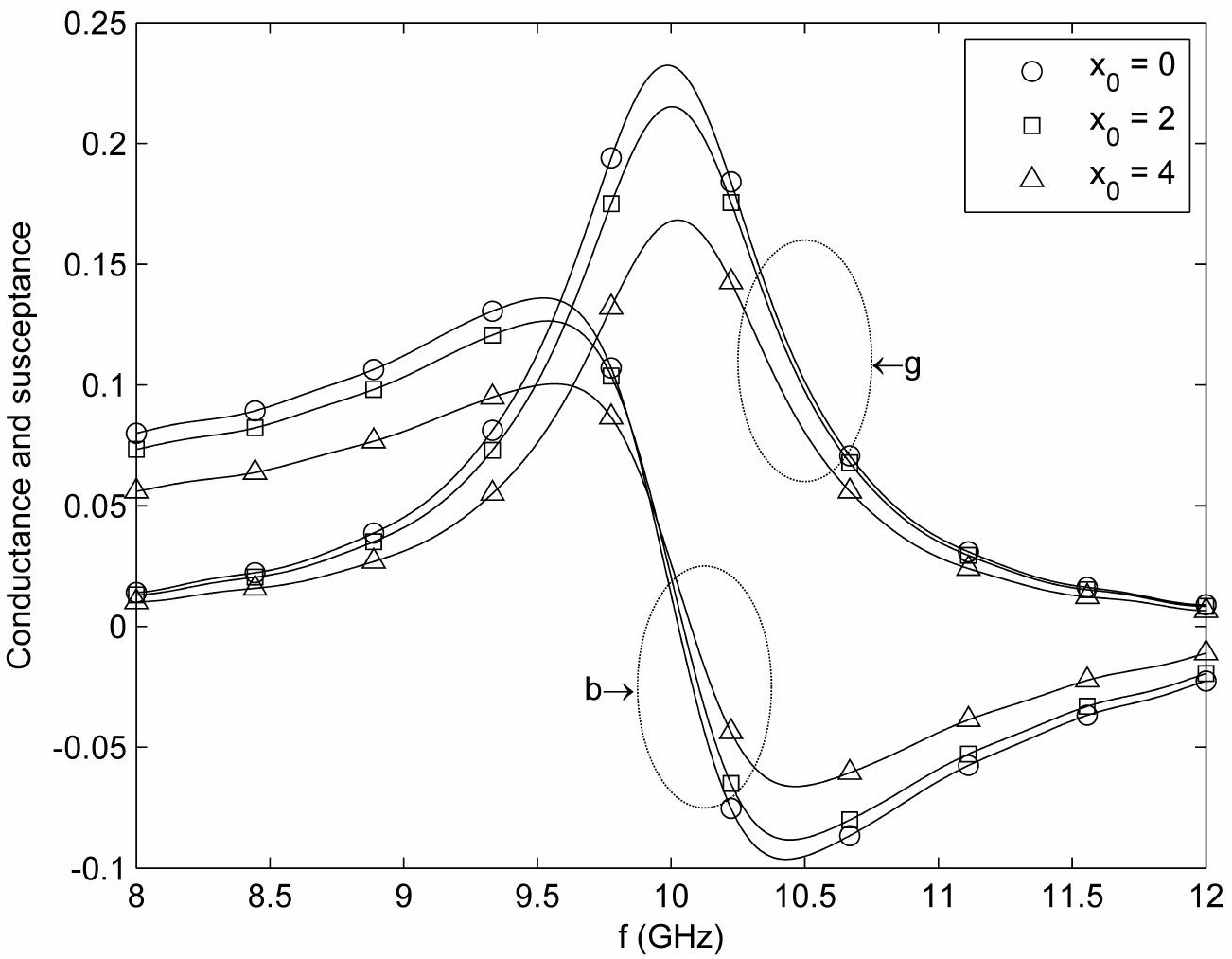




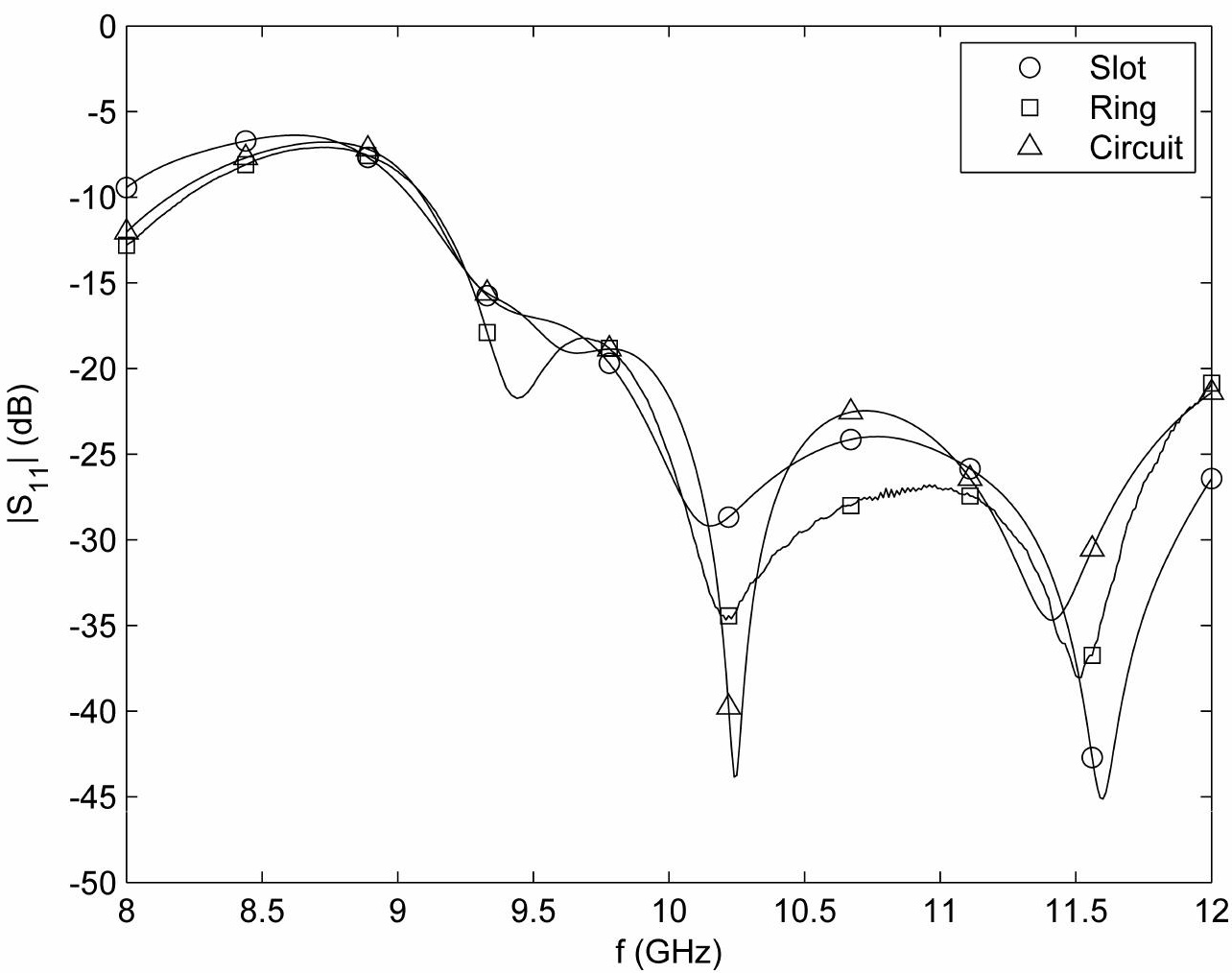




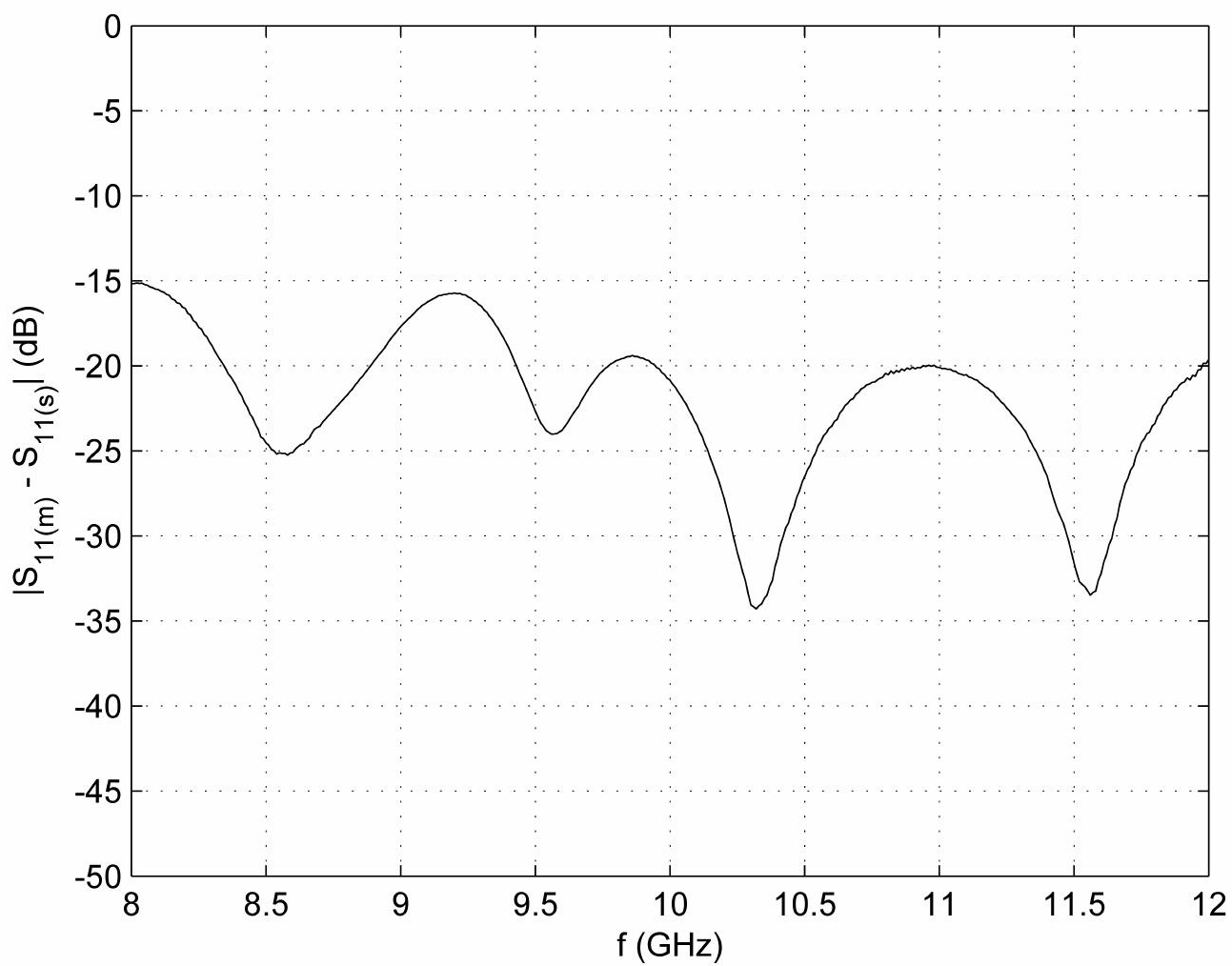

